\title{
On the long term change in the geomagnetic activity during the 20th century
}

\author{
F. Ouattara ${ }^{1}$, C. Amory-Mazaudier ${ }^{2}$, M. Menvielle ${ }^{3,4}$, P. Simon ${ }^{* \dagger}$, and J.-P. Legrand ${ }^{* *}$ \\ ${ }^{1}$ University of Koudougou, BP 376 Koudougou Burkina Faso \\ ${ }^{2}$ LPP - Laboratoire de Physique des Plasmas, CNRS, Université Paris VI - Pierre et Marie Curie, 4 Avenue de Neptune, \\ 94107 Saint-Maur-des-Fossés, France \\ ${ }^{3}$ LATMOS - Laboratoire Atmosphères, Milieux, Observations Spatiales, CNRS, Université Versailles St-Quentin, 4 Avenue \\ de Neptune, 94107 Saint-Maur-des-Fossés, France \\ ${ }^{4}$ Univ. Paris-Sud, Département des Sciences de la Terre, Orsay, France \\ *retired from: Paris's observatory, 5 place Jules Janssen 92190 Meudon, France \\ ** retired from: INSU/CNRS, 3 rue Michel Ange, 75016 Paris, France \\ $\dagger$ deceased 20 January 2008
}

Received: 7 January 2008 - Revised: 18 February 2009 - Accepted: 8 April 2009 - Published: 5 May 2009

\begin{abstract}
The analysis of the aa index series presented in this paper clearly shows that during the last century (1900 to $2000)$ the number of quiet days $(\mathrm{Aa}<20 \mathrm{nT})$ drastically diminished from a mean annual value greater than 270 days per year at the end of the nineteenth century to a mean value of 160 quiet days per year one hundred years later. This decrease is mainly due to the decrease of the number of very quiet days $(\mathrm{Aa}<13 \mathrm{nT})$. We show that the so-evidenced decrease in the number of quiet days cannot be accounted for by drift in the aa baseline resulting in a systematic underestimation of aa during the first quarter of the century: a 2$3 \mathrm{nT}$ overestimation in the aa increase during the 20th century would lead to a $20-40 \%$ overestimation in the decrease of the number of quiet days during the same period.

The quiet days and very quiet days correspond to periods during which the Earth encounters slow solar wind streams flowing in the heliosheet during the period where the solar magnetic field has a dipolar geometry. Therefore, the observed change in the number of quiet days is the signature of a long term evolution of the solar coronal field topology. It may be interpreted in terms of an increase in the magnitude of the solar dipole, the associated decrease of the heliosheet thickness accounting for the observed decrease in the number of quiet days.
\end{abstract}

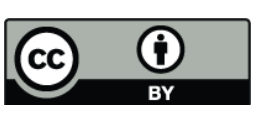

Correspondence to:

C. Amory-Mazaudier

(christine.amory@lpp.polytechnique.fr)
Keywords. Geomagnetism and paleomagnetism (Time variations, secular and long term) - Interplanetary physics (Sources of the solar wind) - Ionosphere (Ionospheremagnetosphere interactions)

\section{Introduction}

The geomagnetic activity is one of the signatures of the Earth's magnetosphere forcing by the solar wind, and it is now well accepted that long data series of geomagnetic indices provide efficient tools for studying the past evolution of solar activity and of its impact on the Earth's environment. The long term geomagnetic data series, and in particular the aa indices (Mayaud, 1968, 1980; Menvielle and Berthelier, 1991) provide continuous information on geomagnetic activity from 1868 onwards, and they are used in a variety of applications ranging from long-term solar and solar wind variability to climate change (e.g., Feynman and Crooker, 1978; Cliver et al., 1998a, b; Lockwood et al., 1999; Solanki et al., 2000; Echer et al., 2004).

One way for characterizing geomagnetic activity and its evolution with time is to study the occurrence of periods during which it becomes low enough for the magnetic situation to be considered as quiet. The identification of such periods of magnetic quietness was actually one of the motivations for defining the very first geomagnetic indices, at the beginning of the twentieth century. At present, planetary geomagnetic

Published by Copernicus Publications on behalf of the European Geosciences Union. 


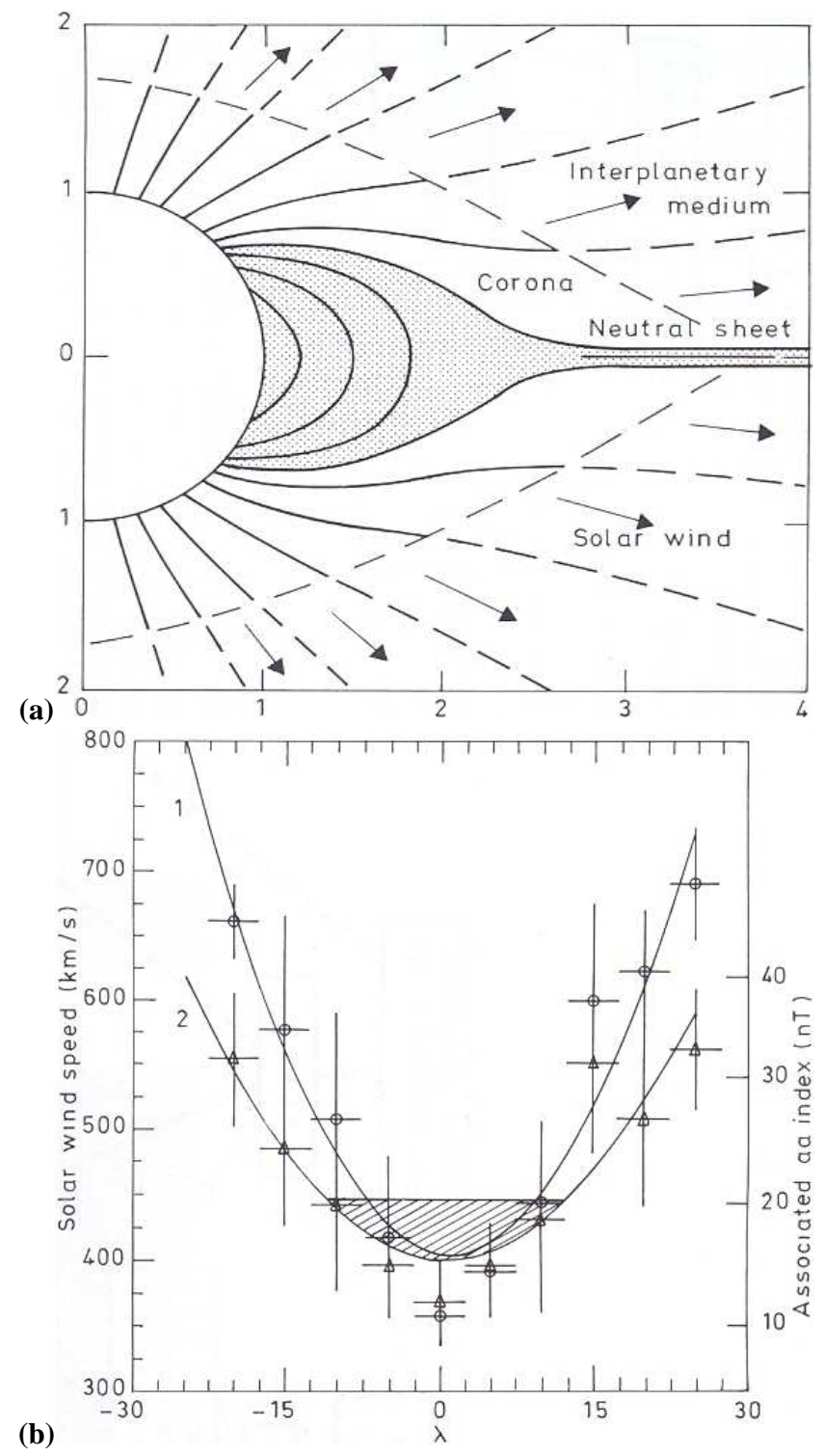

Fig. 1. (a) Model of dipolar field submitted to the internal pressure of the solar plasma flow (Pneuman and Kopp, 1971; reproduced by Simon and Legrand, 1989); (b) Average distribution in the ecliptic plane of the solar wind velocity (scale on the left) in 1976 (circles) and 1977 (triangles) as a function of the angular) in degrees to the neutral sheet (@)distance $(\odot)=0)$ (Bruno et al., 1986). Scale on the right: the velocity associated aa index according to Svalgaard (1977). The hatched area shows the slower solar wind speed $\left(V_{s} \leq 450 \mathrm{~km} \mathrm{~s}^{-1}\right)$ and as a consequence the correlated thickness of its coronal source: the "slow wind sheet".

indices are used to determine periods of magnetic quietness. For instance, many authors define as quiet days those UT days for which the aa daily average (hereafter denoted as Aa indices) is smaller than or equal to a given threshold, e.g., $13 \mathrm{nT}$ (K- and C-days introduced by Mayaud, 1968, 1980, and routinely circulated by the International Service of Geo- magnetic Indices: http://isgi.cetp.ipsl.fr/), or $20 \mathrm{nT}$ (Legrand and Simon, 1989; Simon and Legrand, 1989). In the present study, we use the yearly number of quiet $(\mathrm{Aa}<20 \mathrm{nT})$ and very quiet $(\mathrm{Aa}<13 \mathrm{nT})$ days to characterize the long term evolution of geomagnetic activity from 1868 until now, and infer conclusions on the long term evolution of the solar activity.

The reliability of the results deduced from studies based upon long data series dramatically depends on the homogeneity of the data series. In the particular case of aa, the robustness in the observed increasing trend in the data series, and therefore the validity of the conclusions that rely on this secular change are at present challenged by some authors on the basis of new indices (e.g., Svalgaard et al., 2004; Svalgaard and Cliver, 2007a). These authors argued that there is an inhomogeneity in aa index and thought that aa index needs a new calibration for better utilisation. Many authors addressed the question of the long term homogeneity of the aa data series (see, e.g., Clilverd et al., 2005; Svalgaard and Cliver, 2007b; Lockwood et al., 2007). They concluded that the uncertainty in the aa baseline determination is likely to be on the order of $2-3 \mathrm{nT}$.

Clilverd et al. (2005) also concluded that the robustness of the trend in the aa index supports the idea of a long-term increase in solar coronal magnetic field strength. In particular, they showed that the long term drift that they estimated in the aa baseline cannot account for the observed long-term increase of geomagnetic activity over the 20th century.

In the present study, we use the yearly number of quiet and very quiet days to characterize the long term evolution of geomagnetic activity. We show that these numbers are robust enough to make it possible to derive conclusions on the long term evolution of the solar activity. In Sect. 2 we recall some results concerning solar and geomagnetic activities; Sect. 3 is devoted to the data analysis and the conclusions are summarized in Sect. 4.

\section{Solar phenomena and geomagnetic activity}

In the present paper, we follow the approach proposed by Legrand and Simon (1981) who initiated the use of the geomagnetic index series aa for studying solar activity. They analysed geomagnetic data series over nine solar cycles and studied the dependence of geomagnetic activity to the solar activity.

They identified four classes of magnetic activity that they associated with different solar wind contexts: 1) magnetic quietness due to slow solar wind $(V<450 \mathrm{~km} / \mathrm{h})$ speed escaping from the heliosheet, 2) Shock events resulting from Coronal Mass Ejection, 3) recurrent activity caused by fast solar wind streams from polar coronal holes, and 4) fluctuating activity due to the irregular fluctuations of solar neutral sheet. Since each class of activity refers to solar wind behaviour at the Earth orbit, their long term evolution bears 
information on the long term evolution of solar activity. The reader is referred to Legrand and Simon (1989) and Simon and Legrand (1989) for further details on the definition and occurrence of geomagnetic classes activity.

In this section, we focus on the solar wind conditions associated to magnetic quietness.

Figure 1a (from Pneuman and Kopp, 1971) presents a scheme of the geometry of the solar dipolar magnetic field lines submitted to the internal pressure of the solar plasma flow. The dipolar magnetic field controls the solar wind. Many authors studied the links between the distribution of the wind velocity and the neutral sheet (Borrini et al., 1981; Feldman et al., 1981; Gosling et al., 1981; Zhao and Hundhausen, 1981, 1983; Hoeksema, 1984; Newkirk and Fisk, 1985; Bruno et al., 1986). Bruno et al. (1986) showed that the solar wind velocity is minimal at the neutral sheet, and increases with increasing distance to it; it has an almost symmetric behaviour with respect to the neutral sheet (Fig. 1b). This result is confirmed by Ulysses observations, and it is now well established that magnetic quietness corresponds to slow solar wind flows. Figure $1 \mathrm{~b}$ shows that the $20 \mathrm{nT}$ threshold used by Legrand and Simon to define magnetic quietness corresponds to a solar wind velocity $V_{S}$ of $\sim 450 \mathrm{~km} \mathrm{~s}^{-1}$, namely the "slow solar wind" according to Svalgaard (1977). The width of the slow solar wind layer increases with decreasing intensity of the solar dipolar magnetic field, and accordingly with decreasing solar activity.

These results lead to the following:

1. The thickness of the Heliosheet is related to the solar poloidal magnetic component i.e. a change in the heliosheet thickness is related to a change of the solar magnetic dipole component which regulates $91.5 \%$ of the geomagnetic activity (slow solar wind, high wind speed solar wind and fluctuating activity). The missing $8.5 \%$ of geomagnetic activity is related to CME, which occurred mainly around the sunspot solar maximum (toroidal magnetic component of the sun).

2. A change in the poloidal component of the solar magnetic field impact on the solar wind velocity at the orbit of the Earth.

Kunetsova and Tsirulnik (2008) analysed the solar wind velocity near the Earth's orbit for the period 1964-1997 and found that the trend in the solar wind speed $V$ demonstrates a $55 \%$ increase in the solar wind velocity for the period 1964 1997. Besides, Svalgaard and Cliver (2007b) showed that the yearly mean solar wind speed varied from a low (inferred) of $303 \mathrm{~km} / \mathrm{s}$ in 1902 to a high (observed) value of $545 \mathrm{~km} / \mathrm{s}$. The increase of the solar wind speed $V$ implies also an increase of the electric field $V \times B_{i},\left(B_{i}\right.$ : interplanetary magnetic field $)$ transferred continuously to the magnetosphere by the solar wind / magnetosphere dynamo process (Axford and Hines, 1961) by the solar wind flowing around the magnetospheric cavity. The increase of the $V \times B_{i}$ electric field gives rise to

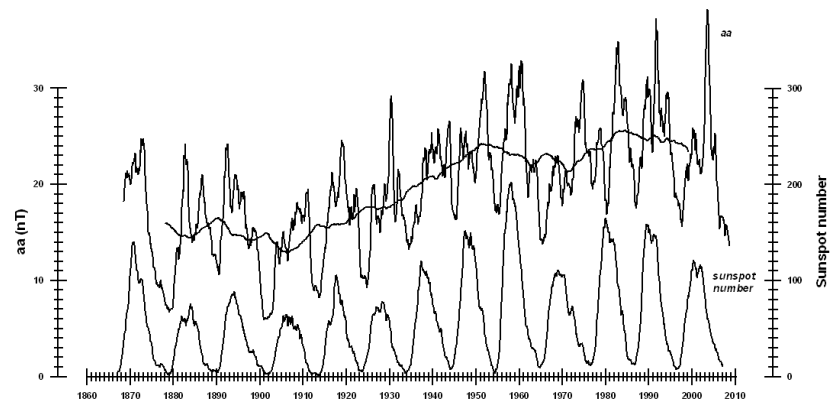

Fig. 2. Long-term variations of aa indices (12-month and 20-year running averages; scale on the left) and of sunspot numbers (12month running averages; scale on the right) from 1868 until now.

an increase in the Energy transferred from the solar wind to the Earth environment.

A magnetic quiet day corresponds to a UT day during which the Earth is most of the time inside the slow solar wind layer. The number of magnetic quiet days accordingly increases with an increasing average width of the slow solar wind layer and, thus, with decreasing solar activity. The long term evolution of the yearly number of magnetic quiet days, therefore, monitors the long term evolution of the solar activity. It is worth noting here that the difference between the origin of magnetic quietness (crossings of the slow solar wind layer by the Earth along its orbit) and magnetic activity (change in the solar wind parameters because of solar events) results in a difference between statistical properties of the distribution of quiet and disturbed days. In particular, as shown in the next section, the yearly number of quiet days is robust with regard to the uncertainty about few $\mathrm{nT}$ in the long term variation of the aa data series.

\section{Long term change in geomagnetic activity}

Figure 2 presents the 12-month running average curve of the aa indices from 1868 until now, together with the 12month running variations of the sunspot number (the socalled smoothed sunspot number). This figure clearly illustrates the behaviour of the geomagnetic activity during the 11-year sunspot cycle. In both aa and sunspot data series, undecennial (11-year) minima occur either simultaneously or with one year difference, but there is no correlation between the values of the minima in both curves. Peaks in the geomagnetic activity are more or less clearly observed almost simultaneously with the undecennial (11-year) sunspot maxima, and they are followed by another peak during the descending phase of each sunspot cycle. The relative intensity of these two peaks varies from one cycle to the other: the peak during the descending phase actually corresponds to the recurrent activity that is generated by solar phenomena related to the forthcoming sunspot solar cycle (see, e.g., Legrand and Simon, 1991). 


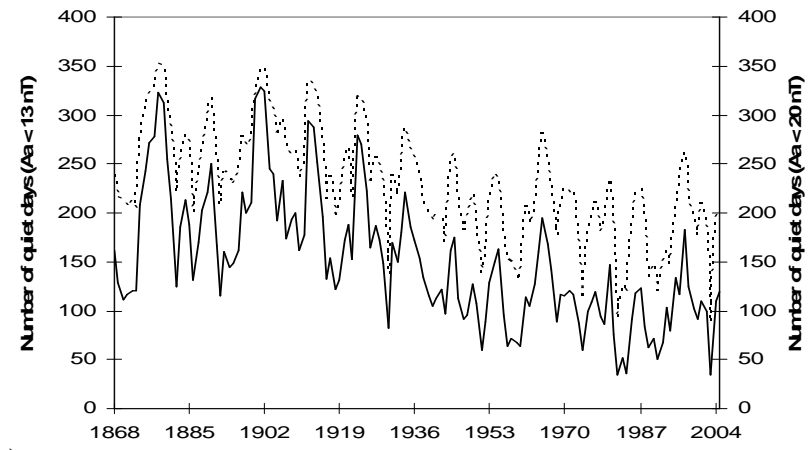

(a)
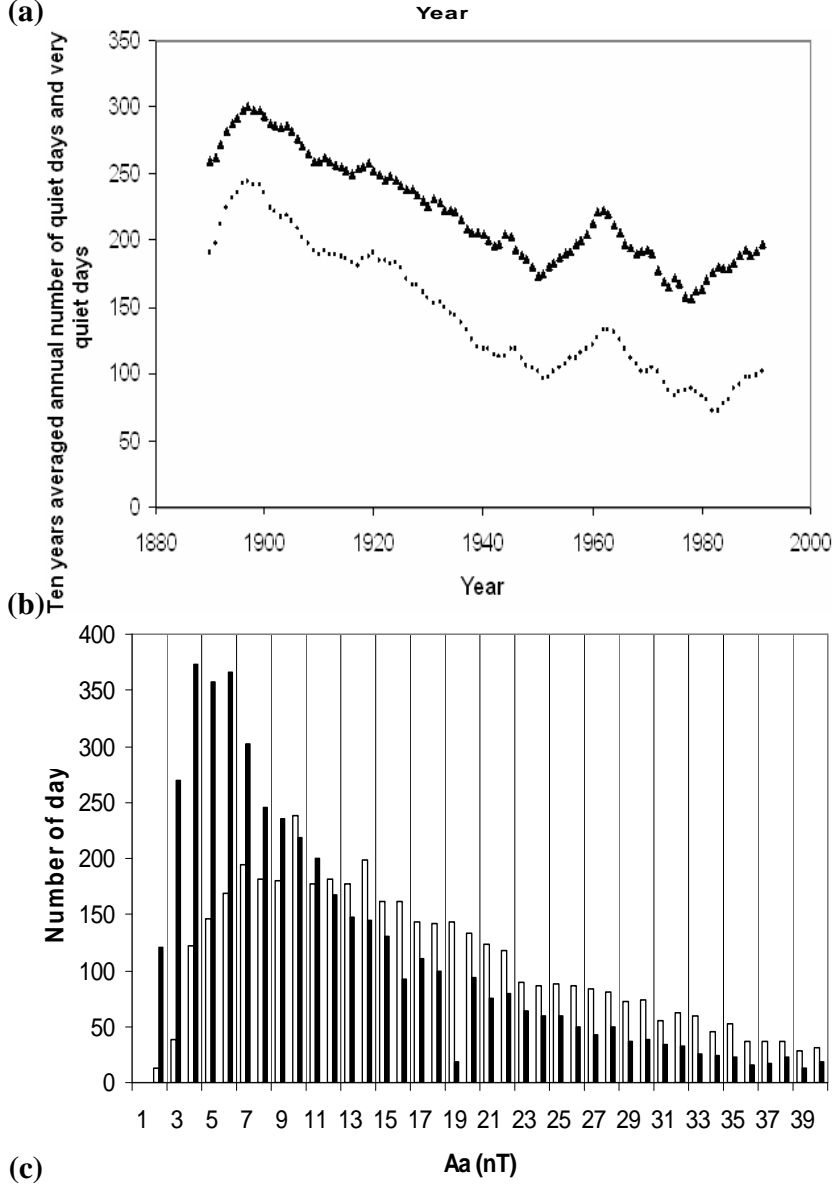

Fig. 3. (a) Annual number of quiet days $(\mathrm{Aa}<20 \mathrm{nT}$; dashed line) and very quiet days $(\mathrm{Aa}<13 \mathrm{nT}$; full line) from 1868 until now; (b) 10-year running average of the number of quiet days (triangles line) and very quiet days (dotted line) with a sliding step of one year; (c) histograms of the Aa values $(0<\mathrm{Aa}<40 \mathrm{nT})$ during solar cycles 12 (black bars) and 20 (white bars).

Figure 2 also highlights a global increase in the level of the geomagnetic activity as described by the aa indices: it is clearly seen when comparing the level of the activity during the first and last thirty years of the data series. The long term trend of this increase is clearly illustrated by the 10 -year running (because solar cycle period is $\sim 10$ or $\sim 11$ years) average curve, also presented on Fig. 2.
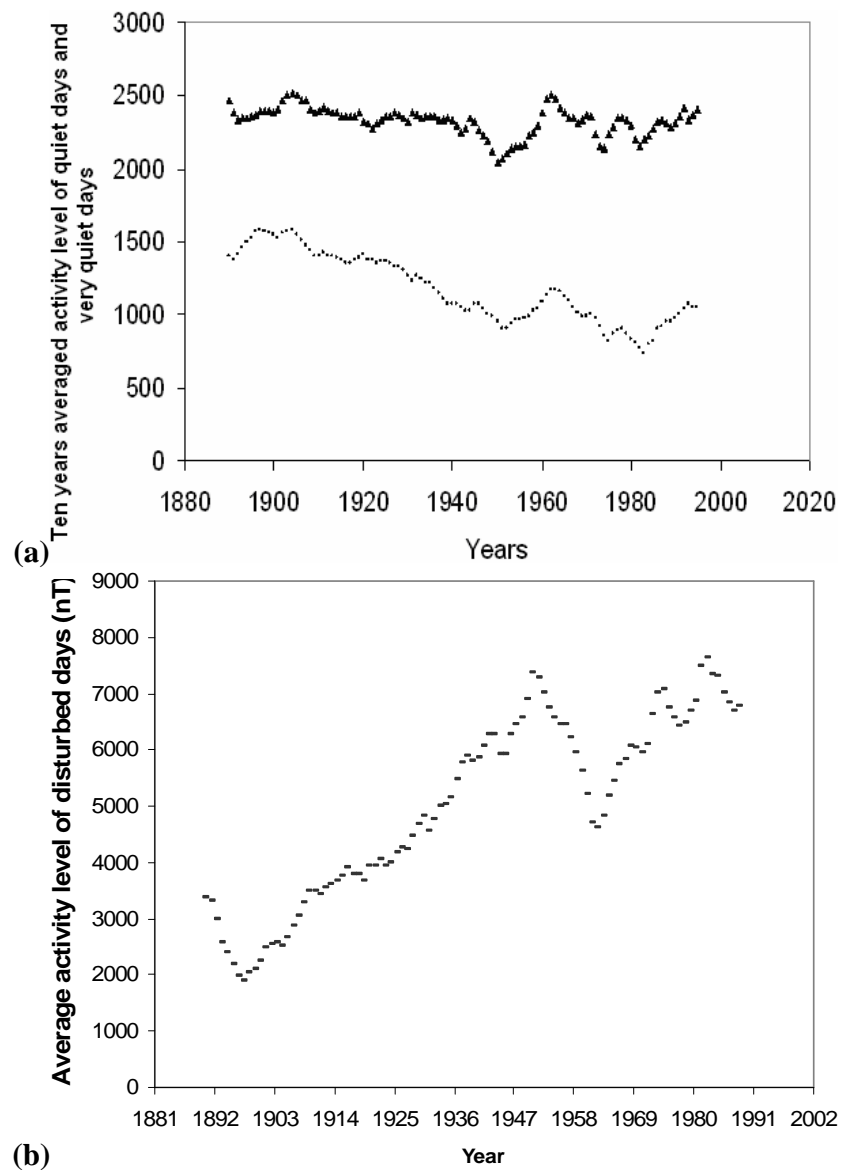

Fig. 4. (a) 10-year running average of the sum of the daily indices during quiet days $(\mathrm{Aa}<20 \mathrm{nT}$; line of triangles) and very quiet days (Aa $<13 \mathrm{nT}$; dotted line), with a sliding step of one year; (b) 10-year running average of the sum of the daily indices during disturbed days $(\mathrm{Aa} \geq 20 \mathrm{nT})$, with a sliding step of one year.

As already stated, the existence of such a long-term increase in the geomagnetic activity is challenged by some authors. We, therefore, consider another quantity, the yearly number of the quiet days, in order to get a robust enough monitoring of the long term variation of the geomagnetic activity from the aa indices data series.

In Fig. 3a are plotted the variations of the annual number of quiet days ( $\mathrm{Aa}<20 \mathrm{nT}$; dotted curve) and very quiet days $(\mathrm{Aa}<13 \mathrm{nT}$; continuous curve) from 1868 until now. As expected, the number of very quiet days is minimum when the geomagnetic activity is maximum (e.g., 1931, 1952, 1957, 1981, 2003), and it is maximum when the activity is minimum (e.g., 1878, 1902, 1913, 1996); it significantly decreases during the twentieth century. A similar behaviour is observed for the number of quiet days; however, the decrease observed in the number of very quiet days is significantly greater than the one observed in the number of quiet days. 
Figure 3a also shows the decrease in the number of quiet days at solar minima during the last century. Figure $3 \mathrm{~b}$ shows the 10-year running average of the numbers of quiet days (triangles) and very quiet days (dots) with a sliding step of one year. The two curves have the same behaviour and clearly illustrate the decrease of the number of quiet and very quiet days. The number of quiet days decreases from more than 270 per year in 1900 to less than 160 per year in 2000; the same trend is observed in the number of very quiet days that decreases from more than 220 in 1900 to less than 75 in 2000.

Let us now assess the robustness of this number with regard to the uncertainty on the long term aa variation, estimated to 2-3nT (see above).

Consider the observed empirical histograms of the Aa indices during each solar cycle. It varies from one cycle to the other, but it is always a unimodal one that peaks at a values smaller than $13 \mathrm{nT}$. Figure $3 \mathrm{c}$ shows the histogram of Aa values during solar cycle 12 (maximum in December 1883, black colour) and solar cycle 20 (maximum in November 1968, white colour). Overestimating by $2 \mathrm{nT}$ the increase in the aa value between solar cycles 11 and 20 leads to underestimating by about 25 days (i.e. $\sim 23 \%$ ) the change in the yearly number of quiet days between these two solar cycles; overestimating by $3 \mathrm{nT}$ this increase reduces the difference in the number of quiet days between cycles 11 and 20 by about 40 days (i.e. less than $40 \%$ of the observed change). Therefore, the decrease observed in the number of very quiet days during the 20th century cannot be accounted for by inconsistency of the long term aa.

Another point of interest is the integral of the aa indices over the set of quiet, very quiet, or disturbed days. Let us calculate for each year the quiet and disturbed components of the geomagnetic activity defined as:

Aaq $=\Sigma$ Aa (quiet days; $\mathrm{Aa} \leq 20 \mathrm{nT}$ )

$\mathrm{Aad}=\Sigma \mathrm{Aa}$ (disturbed days; $\mathrm{Aa}>20 \mathrm{nT}$ )

and also the component of the Aa corresponding to very quiet days:

Aavq $=\Sigma \mathrm{Aa}($ very quiet days; $\mathrm{Aa}<13 \mathrm{nT})$

Figure 4a shows the 10-year running average of Aaq (triangles) and Aavq (dots), for the period 1868 to 2000 with a sliding step of one year. As already noticed by Legrand and Simon (1981), Aaq does no depend on the number of quiet days: it remains quasi constant around $2400 \mathrm{nT}$, except for the period around 1950 where it decreases down to around $2000 \mathrm{nT}$. On the contrary, Aavq almost regularly decreases from $\sim 1500 \mathrm{nT}$, in 1868 (161 very quiet days) to $\sim 1000 \mathrm{nT}$ in 2000 (92 very quiet days) that corresponds to an almost constant $(\sim 10 \mathrm{nT})$ average Aa value per very quiet day.

Table 1 gives the years for which Aaq is smaller than $2000 \mathrm{nT}$ or Aaq is smaller than $1800 \mathrm{nT}$. This table shows that the years for which Aaq is smaller than $2000 \mathrm{nT}$ are in majority after 1950 (16 over 20), while the years for which Aaq is smaller than $1800 \mathrm{nT}$ are in majority after 1960 (7 over 9). This is consistent with an increase of magnetic activity during the twentieth century. Note that the year 2003
Table 1. Years from 1868 until now with Aaq values smaller than $2000 \mathrm{nT}$ (column 2) and $1800 \mathrm{nT}$ (column 4); columns 1 and 3 are years (see text for further explanations).

\begin{tabular}{cccc}
\hline Year & $\mathrm{Aa}_{\text {quiet }}<2000 \mathrm{nT}$ & Year & $\mathrm{Aa}_{\text {quiet }}<1800 \mathrm{nT}$ \\
\hline 1901 & 1840 & 1930 & 1571 \\
1902 & 1980 & 1959 & 1689 \\
1930 & 1571 & 1960 & 1687 \\
1943 & 1967 & 1974 & 1521 \\
1951 & 1804 & $\mathbf{1 9 8 2}$ & $\mathbf{1 4 1 1}$ \\
1952 & 1809 & 1983 & 1696 \\
1956 & 1950 & 1984 & 1795 \\
1957 & 1991 & 1991 & 1739 \\
1958 & 1954 & $\mathbf{2 0 0 3}$ & $\mathbf{1 2 0 7}$ \\
1959 & 1689 & & \\
1960 & 1687 & & \\
1973 & 1903 & & \\
1974 & 1521 & & \\
$\mathbf{1 9 8 2}$ & $\mathbf{1 4 1 1}$ & & \\
1983 & 1696 & & \\
1984 & 1795 & & \\
1991 & 1739 & & \\
1993 & 1989 & & \\
1994 & 1901 & & \\
$\mathbf{2 0 0 3}$ & $\mathbf{1 2 0 7}$ & & \\
\hline
\end{tabular}

corresponds to the smallest value of Aaq over the whole aa data series (see Table 1), but not to the smallest number of quiet or very quiet days (Fig. 3) this suggests that Aa values for very quiet days are particularly low during this year.

Figure $4 \mathrm{~b}$ shows the 10 -year running mean value of the sum of the daily disturbed component (Aad) for the period 1868 until now, with a sliding step of one year. From 1868 until now Aad increases from $\sim 2200 \mathrm{nT}$ until $\sim 7000 \mathrm{nT}$, with a decrease down to $\sim 4600 \mathrm{nT}$ around years $1950-1958$.

In our opinion, these results provide clear evidence for the change in the dipole field independently of the 11-year sunspot variation. This change has been shown by Rouillard et al. (2007). In fact, they show that a mean interplanetary magnetic field strength increases and makes solar wind speed grow by $14 \% \pm 0.7 \%$. Moreover, these authors affirm that on annual timescales, the accumulation of open field lines in coronal holes should force lower expansion rates of magnetic flux tubes. This, in turn, should increase the probability of the Earth intersecting the fast solar wind, thereby raising the average measured solar wind speed. Consequently Rouillard et al. (2007) highlight the decreasing of quiet days number. The above quoted Svalgaard and Cliver (2007b) results on the increase of the solar wind speed over the 20th century are consistent with the observed decreasing of the quiet days number during the 20th century. 


\section{Conclusion}

In this paper, we analyzed the occurrence of magnetic quiet days $(\mathrm{Aa}<20 \mathrm{nT})$ and very quiet days $(\mathrm{Aa}<13 \mathrm{nT})$ during the whole Aa indices data base from 1868-2007. During the last century 1900-2000 a large decrease of the number of magnetic quiet days and very quiet days is observed. Magnetic quiet days decreased from a mean annual value of 270 days per year to a mean annual value of 160 days per years. This strong decrease is mainly due to the decrease of very quiet days. It is a true phenomenon, since the potential effect due to drift in the Aa indices is not likely to account for more than $40 \%$ of the observed decrease.

Magnetic quiet and very quiet days occurred when the Earth is under the influence of slow solar wind speed $(V<450 \mathrm{~km} / \mathrm{s}$ ) flowing out of the Sun from the Heliosheet. Therefore, the decrease of the number of magnetic quiet and very quiet days is the signature of a change in the heliosheet thickness. The larger the heliosheet thickness is, the larger the number of magnetic quiet and very quiet days is (see Fig. 12, bottom panel from Simon and Legrand, 1989).

This paper demonstrates the importance of the analysis of the Earth's geomagnetic activity which is directly related to the evolution of the large scale magnetic components of the solar magnetic field.

Acknowledgements. The sunspot numbers used in this study are provided by the World Data Center for the Sunspot Index, Royal Observatory of Belgium, Monthly Report on the International Sunspot Number, online catalogue of the sunspot index: http: //www.sidc.be/sunspot-data/; the aa indices are provided by the International service of Geomagnetic Indices, Centre d'études des Environnements Terrestre et Planétaires, http://isgi.cetp.ipsl.fr/.

Topical Editor M. Pinnock thanks F. Lefeuvre and two other anonymous referees for their help in evaluating this paper.

\section{References}

Borrini, G. T., Gosling, J. T., Bame, S. J., Feldman, W. C., and Wilcox, J. M.: Solar wind helium and hydrogen structure near the heliospheric current sheet : a signal of coronal streamee at 1 AU, J. Geophys. Res., 86, 4565-4573, 1981.

Bruno, R., Villante, U., Bassano, B., Schwenn, R., and Mariani, F.: In observations of the latitudinal gradients of the solar wind parameters during 1976 and 1977, Solar Phys., 104, 432-445, 1986.

Clilverd, M. A., Clarke, E., Ulich, T., Linthe, J., and Rishbeth, H.: Reconstructing the long-term aa index, J. Geophys. Res., 110, A07205, doi:10.1029/2004JA010762, 2005.

Cliver, E. W., Boriakoff, V., and Bounar, K. H.: Geomagnetic activity and the solar wind during the Maunder Minimum, Geophys. Res. Lett., 25(6), 897-900, 1998.

Cliver, E. W., Boriakoff, V., and Feynman, J.: Solar variability and climate change: Geomagnetic AA index and global surface temperature, Geophys. Res. Lett., 25(7), 1035-1038, 1998.

Echer, E., Gonzalez, W. D., Gonzalez, A. L. C., Prestes, A., Vieira, L. E. A., Dal Lago, A., Guarnieri, F. L., and Schuch, N. J.: Long- term correlation between solar and geomagnetic activity, J. Atmos. Solar-Terr. Phys., 66(12), 1019-1025, 2004.

Feldman, W. C., Asbridge, J. R., Bame, S. J., Fenimore, E. E., and Gosling, J. T.: The solar origin of solar wind interstream flows : near equatorial coronal streamers, J. Geophys. Res., 86, 54085416, 1981.

Feynman, J. and Crooker, N. U.: The solar wind at the turn of the century, Nature, 275, 626-627, 1978.

Golsing, J. T., Borrini, G., Asbridgee, J. R., Bame, S. J., Feldman, W. C., and Hanse, R. T.: Coronal streamers in the solar wind at 1 AU, J. Geophys. Res., 86, 5438-5448, 1981.

Hoeksema, J. T.: Structure and evolution of the large scale solar and heliopheric magnetic fields, Thesis CSSA-ASREO-84, Center for Space Science and Asstrophysics, Standford U., California, 1984.

Kuznetsova, T. and Tsirulnik, L. B.: Time change of solar activity, interplanetary magnetic field and solar wind velocity at the earth's orbit in different spectral bandes, J. Atmos. Solar Terr. Phys., 70, 254-260, 2008.

Legrand, J. P. and Simon, P. A.: Ten cycles of solar and geomagnetic activity, Solar Phys., 70, 173-195, 1981.

Legrand, J.-P. and Simon, P. A.: Solar cycle and geomagnetic activity : A review for geophysicists Part I : the contribution to geomagnetic activity of shock waves and of the solar wind, Ann. Geophys., 7(6), 565-578, 1989.

Legrand, J. P. and Simon, P. A.: A two component solar cycle, Solar Phys., 131, 187-209, 1991.

Lockwood, M., Stamper, R., and Wild, M. N.: A Doubling of the Sun's Coronal Magnetic Field during the Last 100 Years, Nature, 399, 437-439, 1999.

Mayaud, P. N.: A hundred year series of geomagnetic data, 18681967: indices aa, storm sudden commencements, IAGA Bulletin, 33, 256 pp., IUGG Publ. Office, Paris, 1973.

Mayaud, P. N.: Derivation, meaning and use of geomagnetic indices, AGU Geophys. Monogr., 22, 154 pp., AGU, Washington, USA, 1980.

Menvielle, M. and Berthelier, A.: The K-derived planetary indices: description and availability, Rewiews Geophys.Space Phys., 29, 415-432, (erratum, Rewiews Geophys.Space Phys., 30, 91), 1991.

Newkirk, G. and Fisk, A.: Variations of cosmic rays and solar wind properties with respect to the heliospheric current sheet, 1 fiveGeV protons and solar wind, J. Geophys. Res., 90, 3391-3414, 1985.

Pneuman, G. W. and Kopp, R. A.: Gas-magnetic field interactions in the solar corona, Solar Phys., 18, 258-270, 1971.

Rouillard, A. P., Lockwood, M., and Finch, I.: Centennial changes in the solar wind speed and in the open solar flux, J. Geophys. Res., 112, A05103, doi:10.1029/2006JA012130, 2007.

Simon P. A. and Legrand, J. P.: Solar cycle and geomagnetic activity : A review for geophysicists Part II. The solar sources of geomagnetic activity and their links with sunspot cycle activity, Ann. Geophys., 7(6), 579-594, 1989.

Solanki, S. K., Schüssler, K., and Fligge, M.: Evolution of the Sun's large-scale magnetic field since the Maunder Minimum, Nature, 408(6811), 445-447, 2000.

Svalgaard, L.: Geomagnetic activity : dependence on solar wind parameters, in: Coronal holes and high speed wind streams, edited by: Zirker, J. B., Colorado Ass. Univ. Press, Boulder, 371-432, 
1977.

Svalgaard, L., Cliver, E. W., and Le Sager, P.: IHV: A new longterm geomagnetic index, Adv. Space. Res., 34, 436-439, 2004.

Svalgaard, L. and Cliver, E. W.: A floor in the solar wind magnetic field, The Astrophys. J., 661, L203-L206, 2007a.

Svalgaard, L. and Cliver, E. W.: Interhourly variability index of geomagnetic activity and its use in deriving the long-term variation of solar wind speed, J. Geophys. Res., 112, A10111, doi:10.1029/2007JA012437, 2007b.
Zhao, X.-P. and Hundhausen, A. J.: Organization of solar wind plasma properties in a tilted heliomagnetic coordinate system, J. Geophys. Res., 86, 5423-5340, 1981.

Zhao, X.-P. and Hundhausen, A. J.: Spatial structure of solar wind in 1976, J. Geophys. Res., 88, 451-454, 1983. 